\title{
RFID BASED SMART TROLLEY
}

\author{
Dheepika Raajha \\ Department of E\&TC \\ PCCOE, Pune, Maharashtra, India
}

\author{
Aditi Kurankar \\ Department of E\&TC \\ PCCOE, Pune, Maharashtra, India
}

\begin{abstract}
With the upsurge in wireless technologies, the necessity for an ultimatum for a facile and swift bill payment has risen in supermarkets. In this paper, we canvass an ingenious concept of intelligent shopping and smart billing. To overcome the problems in the existing system, RFID tags are attached to all the products in the supermarkets for identification. Each shopping cart is implemented with a Product Identification Device (PID) that contains a AT89S52 microcontroller, 16x2 LCD, RC522 RFID module and a Tarang F4 ZIGBEE transmitter. The information of the purchased products on the shopping cart are read by the RFID reader and is displayed on liquid crystal display, which is interfaced to the microcontroller. Using ZIGBEE module, the total bill will be transferred to the computer at the counter side along which a SMS of the receipt is also sent to the customer. As the entire framework becomes smart the labor requirement will decrease along these lines. Subsequently, benefiting the customers and retail merchants as more customers can be served in the same time.
\end{abstract}

Keywords- RFID, ZIGBEE, PID

\section{INTRODUCTION}

The break off of wireless technologies along with other communication techniques has played a significant role in making e-commerce a popular choice. Contemporary innovative products assist in improvising the comfort, convenience and efficiency of day to day activities. In this project, we use an innovative concept of RFID Based Smart Shopping and Billing System. The principal aim is to aid shopping in person, thus providing an economical technology oriented, easily scalable, and robust system. The smart shopping cart will help shorten the checkout lines thereby improvising the customer experience at supermarkets. The system consists of an RFID based trolley which communicates with the billing counter wirelessly using a ZigBee Transmitter. The trolley will have a similar type of hardware with a unique address for each trolley. The developed system constitutes of a User Interface and Display Unit (UIDU). The products can be scanned by the customers themselves simultaneously the total amount will updated on the LCD screen of the smart trolley. Until he goes to the counter for paying the bill, the customer is not aware of the amount of total purchase in the current system. Once the customer approaches the counter, products not required can be reverted back. To curtail these issues, we introduce the concept of a smart trolley, where the total bill of the purchased products can be checked out beforehand, with the bill amount also being sent to the billing section wirelessly. Thereby benefiting the emporiums as more people will enjoy the shopping experience and visit more frequently.

\section{EXISTING SYSTEM}

The existing system of shopping is a tiresome, prolonged process which includes choosing the products, scanning them, waiting in queues and checking out. Often time is wasted in incessant queues and billing of the merchandise which creates a mayhem in the shopping malls. We could rather use the trending cutting edge technologies to cut short the time taken and resolve the problem. In the barcode method that is currently employed in supermarkets, every product is labelled with a barcode sticker which may be browsed through specially designed barcode readers which consist of a lightweight supply, a light sensor and lens that translate optical impulses into electrical signals. Additionally, decoder circuitry is present in nearly all the barcode readers to analyze the barcode's image data provided by the sensor and to send the barcode's content to the scanner's output port. After purchasing a product, we have a tendency to place it in the trolley and take it to the counter. After which, the cashier scans the merchandise through the barcode scanner and offers the bill. But this becomes a painstaking process when tons of merchandise are to be scanned, eventually resulting in long queues.

\section{PROPOSED SYSTEM}

Radio frequency identification, a computerized ID innovation in which radio recurrence waves are used to exchange information between an onlooker and items that have RFID tags attached to them is gradually taking over barcodes. In this paper, we have developed a smart shopping trolley that facilitates management of shopping lists of customers while shopping and only pay the bill at the time of checkout. The trolley has the potential to compute mechanically and display the cost of all the products in it, making it easier for the customer to be aware of the amount he or she has to pay beforehand and not at the time of checkout. This way the customer will receive swifter service at the time of checkout and the shop owners too could reduce the staff. RFID reader is attached to AT89S52 microcontroller. The tag is scanned by the reader and a signal is sent to the controller as the user puts items in 


\section{International Journal of Engineering Applied Sciences and Technology, 2020 \\ Vol. 5, Issue 2, ISSN No. 2455-2143, Pages 520-524 \\ Published Online June 2020 in IJEAST (http://www.ijeast.com)}

the trolley. Data is then stored it in the memory by the controller and compared with the product ID. On tally, the name of item is displayed on the screen along with the total cost of purchase. Once done with shopping, a button can be pressed on the trolley which would send the total amount spent on the products to the billing counter section using ZIGBEE transmitter wirelessly. Products can also be reverted back as per our budget and requirement. When an item is taken back from the trolley, the same card will again be read by the RFID reader after which the same amount associated with product will be deducted by the controller from the net bill amount. The ZIGBEE receiver then sends the bill amount to the computer to display on the hyper terminal along with which a SMS of the receipt is sent to the customer.

\section{WHY RFID?}

Radio Frequency Identification (RFID) is gradually gaining momentum to become the more preferable technology as an alternative to barcode systems. An automatic identification method is provided by RFID systems, relying on storing and remotely retrieving data using RFID tags or transponders. An RFID tag is an associate object that can be connected to or integrated with a product for identification using radio waves and to transfer information from an associate electronic tag, known as RFID tag or label, connected to associate object, through a reader. RFID tags are wireless cards that have a built-in embedded silicon chip alongside loop antenna. A $125 \mathrm{KHZ}$ magnetic signal is generated by the RFID reader which is later transmitted by the loop antenna which scans the RFID card. All the products are equipped with an unique RFID card that indicates the merchandise name thus rendering it to be a secure access card. Microcontroller is interfaced with the RFID reader. Some radical applications of RFID: 1. Authentication 2. Asset tagging and identification 3. Keyless entry 4. Point-of-sale 5. Self check-in and self check-out

\section{HARDWARE}

\section{A. RFID -}

A RFID reader is accustomed to collect information from a RFID tag to trace products. A low-power radio emission field is emanated from a RFID reader powering up the tag to pass data held by the chip. In theory, RFID could be a technology analogous to bar codes. But RFID tags don't necessarily need line-of-sight with the reader, so don't have to be scanned directly. The RFID tag should be in the proximity of a RFID reader, i.e. within 3 - 300 feet, in order to be scanned. RFID technology enables speedy identification of a specific product, even if it is surrounded by various things.

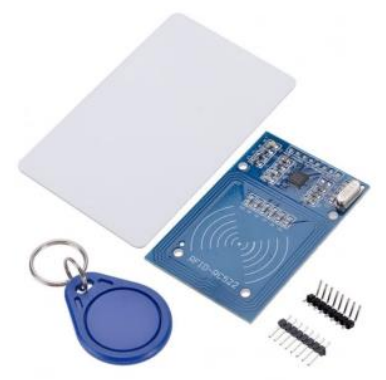

Fig. 1. RFID-RC522 Module

\section{B. LCD Display -}

LCD (Liquid Crystal Display) screen is an electronic display module having extensive applications. A 16x2 display being an fundamental module is the most preferred over seven phases and other multi segment LEDs. They are easily programmable, cost effective and don't have any limitation of displaying special and even custom characters along with animations having a display of two lines of 16 characters each. In this digital display, every character is displayed in a $5 \times 7$ picture element matrix having two registers, viz. information and command.

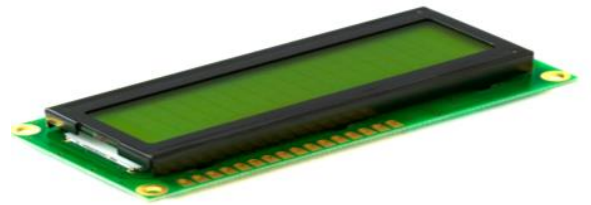

Fig. 2. LCD

\section{Microcontroller -}

The AT89S52 microcontroller is a low voltage, high performance, powerful CMOS 8-bit microcomputer, which is highly flexible and provides a cost effective solution to many embedded control applications by combining a versatile 8-bit control processing unit with a flash on a monolithic chip. Atmel's high density nonvolatile memory technology is used in manufacturing this device. Being compatible with industrystandard MCS-51 instructions set, the on-chip flash will also allow reprogramming of the program memory in the system or by a conventional non-volatile memory programmer. The AT89S52 microcontroller is designed with static logic for operation down to 0 frequency and it will support 2 software selectable energy saving modes. While allowing the RAM, serial port, counter or timer and interrupt system to continue functioning, the idle mode stops control processing unit. While the RAM contents will be saved in the power-down mode, the oscillator will be frozen by disabling all other chip functions until next hardware reset is done. 


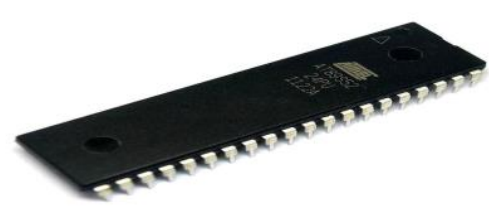

Fig. 3. AT89S52 Microcontroller

\section{ZIGBEE -}

ZIGBEE is a wireless technology evolved in regard with global standards in order to address the distinct requirements of low wattage, cost effective wireless IoT networks that supports low data rates, energy efficiency and secure networking designed to create personal area networks.

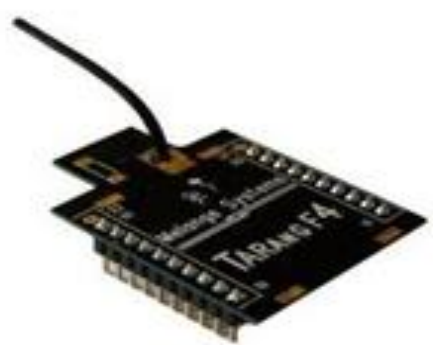

Fig. 4. ZIGBEE Tarang F4

\section{E. MAX232-}

MAX232 IC is a specially designed circuit that dispenses the best noise rejection by making standard voltages as per the requirement of $\mathrm{RS} 232$ standards. To ensure data transfer between microcontroller and PC, the voltage levels and baud rate should be the equal. The voltage levels supported are logic $1(+5 \mathrm{~V})$ and logic $0(0 \mathrm{~V})$. But for a computer, RS232 voltage levels are taken as $-3 \mathrm{~V}$ to $-25 \mathrm{~V}$ for logic 1 and 0 as +3 to $+25 \mathrm{~V}$ for logic 0 . MAX232 IC is used so as to commensurate these voltage levels. Thus these voltage levels are converted to microcontroller voltage levels by IC RS232.

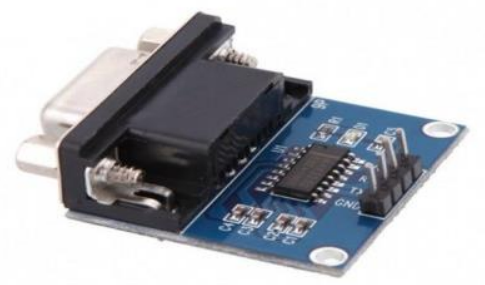

Fig. 5. MAX232

\section{F. GSM -}

Global system for mobile communication is an open and digital cellular technology used for transmitting mobile voice and data services operating at $850 \mathrm{MHZ}, 900 \mathrm{MHZ}, 1800 \mathrm{MHZ}$ and 1900MHZ frequency bands. The idea of GSM was developed in Bell Laboratories in 1970. Time division multiple access (TDMA) technique is employed for communication purposes in this technology. Data is digitalized and compressed before being sent down through a channel with two different streams of user data, each in its own particular time slot.

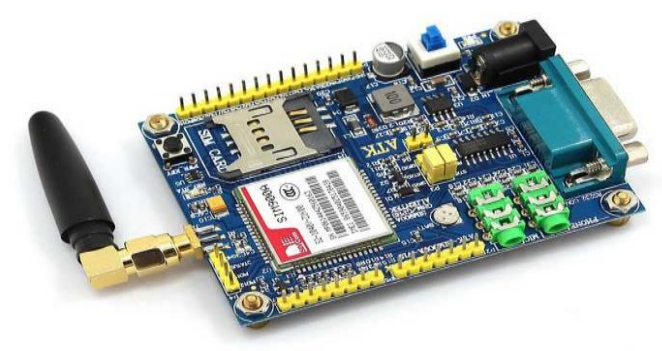

Fig. 6. GSM SIM900A

\section{G. ATMEL 8051 AVR USB ISP PROGRAMMER -}

8051 ISP Programmer is a low cost, easy to use, 10 pin interface USB based in-system programmer that enables PLDs, microcontrollers, and other embedded devices to be programmed while installed in a complete system, rather than requiring the chip to be programmed prior to installing it into the system. Hence allowing you to program your target microcontroller without removing it from your development board. The greatest feature of this board is the ability to power the target (up to $500 \mathrm{~mA}$ ) from the programmer and $5 \mathrm{~KB} / \mathrm{sec}$ maximum write speed.

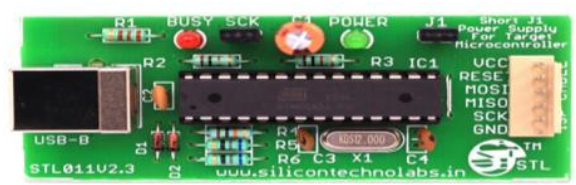

Fig. 7. AVR USB ISP PROGRAMMER 


\section{FLOWHART}

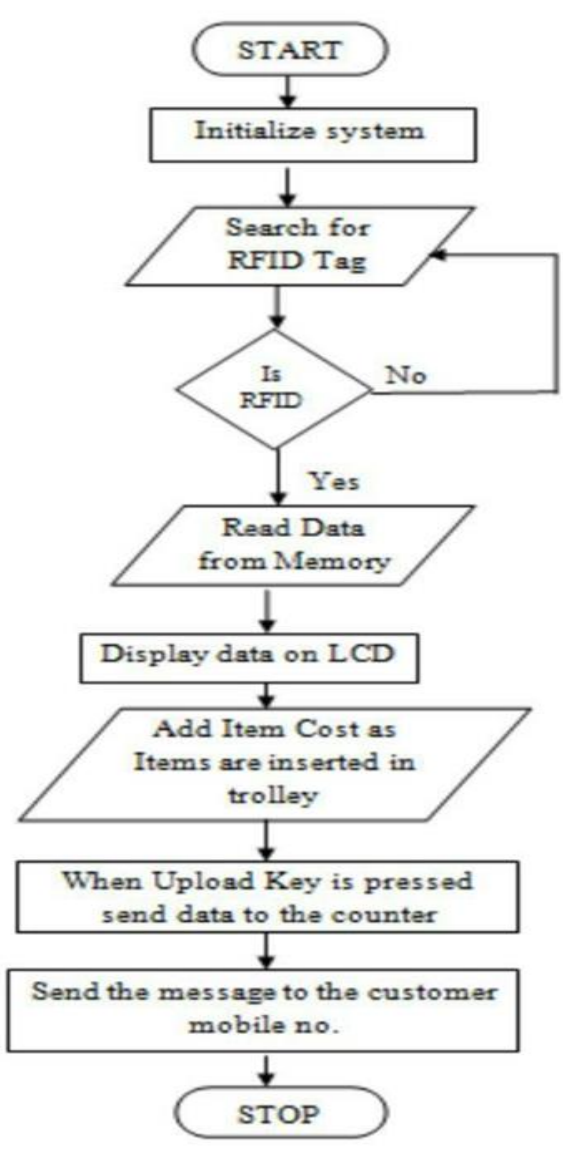

Fig. 8. Flowchart

VII. WORKING

1. Every product in the supermarket will be equipped with RFID cards. The card will be scanned by the RFID reader when a person puts a commodity in the trolley.

2. The code is sent by the reader to the microcontroller which then reads the product's name, price and other details and displays it on the screen.

3. The cost of the products will get added to the total as we add the items in the cart and simultaneously all details are displayed on the liquid crystal display which is a $16 \times 2$ character alphanumeric type display. And additionally, if we wish to discard some inserted item, then that amount is mechanically subtracted from the entire amount and item removal message is displayed on the LCD.

4. Objects are interfaced with transponders. An RFID tag comprises of a very small microchip and antenna, which come in various dimensions, shapes and forms. Wireless communication occurs between the RFID reader and transponders for which a line of sight is not required between the devices. An RFID reader can read through almost everything. Radio waves are emitted by the tags affixed to the products which are encountered by the RFID reader in the trolley system; a magnetic field is emitted by the spiraled antenna in the smart labels. The circuits in the transponder are energized when power is withdrawn by the smart labels. The data present in the smart label is sent by transforming the radio waves to a more utilizable form of data.

5. The liquid crystal display is interfaced with the microcontroller. It is used to indicate the customer's actions i.e. insertion of the product, withdrawal of the product, product's price and the cumulative sum of products in the trolley.

6. The bill is then sent to the customer via SMS.

The block diagram of the proposed system is as shown in fig 9:

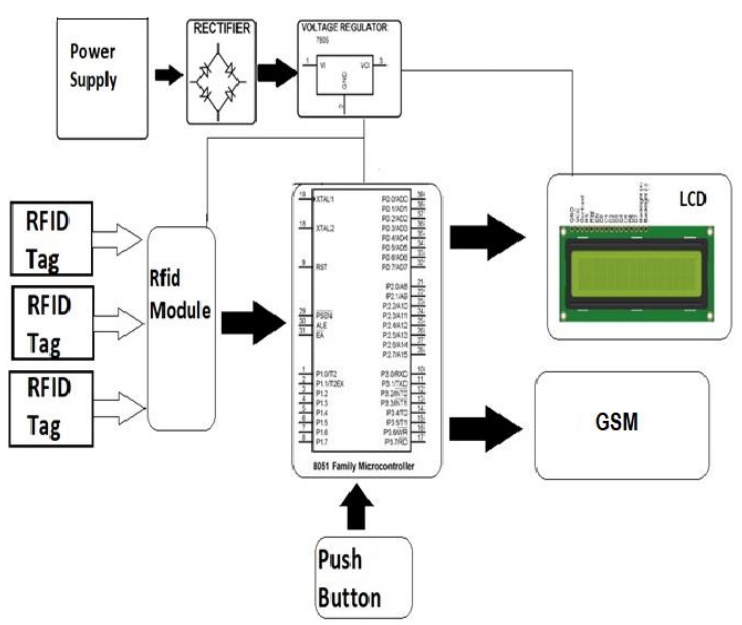

Fig. 9. Block Diagram

1)TROLLEY SECTION - The block diagram of trolley section is as shown in fig 10 :

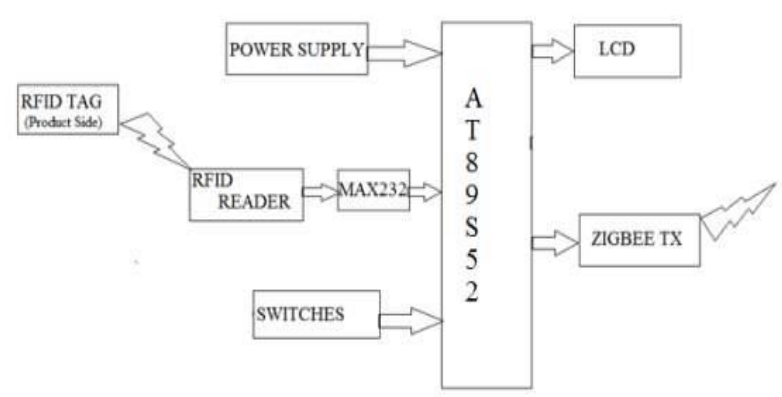

Fig. 10. Trolley Section 
2)COUNTER SECTION - The block diagram of trolley section is as shown in fig 11 :

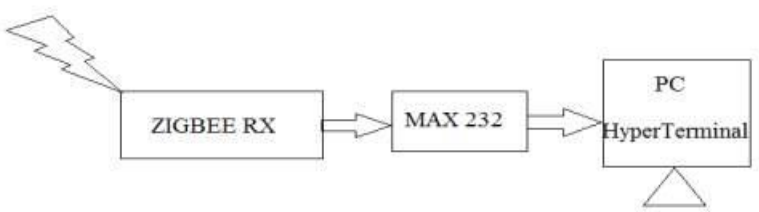

Fig. 11. Counter Section

\section{FUTURE SCOPE}

We can anticipate to effectuate it in supermarkets using a long range RFID reader (with a range of $\sim 2$ meters) in the future. Furthermore, an advanced microcontroller, a module with larger display and net banking can be introduced. Databases can be shifted to cloud and we could move to an IOT platform for faster and more efficient workflow. A virtual assistant to help locate items along with a GPS can be employed. Additionally, robotic ARM could be employed for picking and dropping of products. Thus, delivering improvised services and customer experience with optimum time complexity.

\section{CONCLUSION}

We envisage to modify the billing procedure by making it technology driven, economical, scalable and into a robust system in order to expedite shopping in person. The trolley has the facet to compute mechanically and display the cost of all the products within it, thus making the client aware of what amount he or she has got to pay beforehand rather than at the time of checkout. Additionally, products can be effaced from the trolley by scanning it once more if he or she does not require it. The proposed system is highly reliable, secure, steadfast and cost effective. As the entire framework is becomes smart, the labor requirement will reduce, along these lines profiting the retail merchants. System parameters of smart shopping cart like product name and cost are constantly displayed.

\section{REFERENCES}

[1] Chadha Raghav, Kakkar Srishti, Aggarwal Garima, "Automated Shopping and Billing System Using RadioFrequency Identification", $9^{\text {th }}$ International conference on cloud computing, data science and engineering (IEEE), 2019.

[2] Sammeta Goud Santosh, Madara Reddy Sahith, "Recent trends in RFID technologies and its impact on universities", IEEE Advances in Science and Engineering Technology International Conferences (ASET), 2018.

[3] Kumar Akshay, Gupta Abhinav, S.Balamurugan, S.Balaji, R.Marimuthu, "Smart Shopping Cart", IEEE International conference on Microelectronic Devices, Circuits and Systems, 2017.
[4] Dawkhar Kalyani, Dhomase Shraddha, Mahabaleshwarkar Samruddhi, "Electronic Shopping Cart For Effective Shopping based on RFID”, International Journal of Innovative Research In Electrical, Electronic, Instrumentation And Control Engineering Vol. 3, Issue 1 pp 84-86, January 2015.

[5] Chaudhari Subhash Mayur, "A Review on Electronic Shopping Cart Based on RFID", International Journal and Magazine of Engineering. Technology, Management and Research, 2015.

[6] P. Chandrasekar and T. Sangeetha, "Smart Shopping Cart with Automatic Billing System through RFID and ZigBee ", IEEE, 2014.

[7] Jin-Shyan Lee, Yuan-Ming Wang, "Experimental Evaluation of ZigBee-Based Wireless network in Indoor environment", Journal Of Engineering, 18 Feb 2013.

[8] C.Muthu Ramya, M.Shsnmugaraj, R.Prabhakarn, "Study on ZigBee technology", IEEE 3rd International Conference on Electronics Computer Technology, 2011.

[9] Ma Yuchun, Huang Yinghong, Zhang Kun, Li Zhuang, "General Application Research on GSM Module", IEEE International Conference on Internet Computing and Information Services, 2011.

[10] Molisch F. Andreas, "GSM Global System for Mobile Communications”, Wiley-IEEE Press, pp.587-620, 2011. 